Original Article

Corresponding Author:

Peter Twigg

The School of Engineering

Manchester Metropolitan University

M1 5GD

p.twigg@mmu.ac.uk

\title{
Workshop problem-solving for improved student engagement and increased learning in Engineering Control
}

\author{
Peter Twigg \\ Prasad Ponappalli \\ Margaret Fowler \\ The School of Engineering \\ Manchester Metropolitan University
}

\begin{abstract}
Study of Engineering Control is often perceived by students as highly complex and a difficult hurdle to pass. The authors of this paper were prompted to propose changes to the teaching of a final year control unit of a BEng (Hons) Degree in Electrical and Electronic Engineering after numerous students had expressed concern about their ability to be successful in this subject. The average exam grade had also been low for several years. Teaching previously had been didactic in nature, based on lectures, predefined
\end{abstract}


laboratory exercises and tutorial sessions. Use of appropriate based workshop study sessions can lead to higher levels of attainment and development, and student perception is that the experience is informal, engaging but appropriately challenging. A series of workshop sessions were introduced into term two of the unit teaching over a period of several weeks. Students worked individually on learning improvement activities, within cooperative groups in an informal workshop environment. The tasks set to the students were challenging and designed to improve general skills in Control, and students were encouraged to explore further areas that interested them. Results presented in this paper describe an improvement in student engagement, confidence in exam preparation and communication of learning measured by grades since the introduction of the workshop sessions, and improved feedback from students. The workshop sessions were integrated into the teaching scheme rather than being elective, and did not require any additional timetable sessions.

\section{Keywords}

Workshops, control, engagement, problem-based learning, peer support, cooperative learning 


\section{Introduction}

The degree course in Electrical and Electronic Engineering at Manchester Metropolitan University includes a final year unit on Automation and Control. Previously students had consistently expressed fears about this unit and it had a reputation for being difficult to pass. Teaching staff had observed that some students tended to put off tackling the study for what they considered to be the mathematically and conceptually challenging areas of the unit causing problems for them later in the year. There were many requests for extra tuition and one to one sessions, creating an additional load on teaching staff. Comments from students suggested that they felt that the unit content was too mathematical with insufficient application and did not address realistic problems that a Control Engineer would face. The average final exam mark in the previous five years had been consistently around $52 \%$. An initial response to the students' concerns was to significantly increase the scheduled tutorial support. This did not produce any measurable change to the average exam grade of the students that year, but did cause a further increase in the teaching load for unit staff. Consequently an alternative, workshop-based approach was defined and implemented.

Published studies have indicated that increased workshop activities and reduced lecture time can improve student feedback, improve student engagement and allow students to score higher in exams ${ }^{[1]}$; workshops established for introductory degree courses have been set up for students to work in small co-operative groups to solve challenging problems, evaluate case studies and engage in activities designed to improve general learning skills. A number of studies also indicate that activities designed to increase peer and instructor discussions increase engagement and learning ${ }^{[2]}$. Research carried out in Peer-Led Team Learning has shown that there tends to be more knowledge exchanged amongst students working in co-operative groups compared to that between lecturer and student ${ }^{[3]}$; statistical results showed significant improvements in student performance and attitude towards a course. In studies where elective supplemental programmes have been introduced, those students who chose to attend the supplemental programmes achieve higher grades than those students who did not, but that most students chose not to attend, ${ }^{[4]}$. Programmes that embed effective study learning practices into the course structure have the highest likelihood of improving student performance and retention, ${ }^{[5]}$, addressing the imperative for institutions to avoid wasting time and resources on activities that do not contribute to students' success. Didactic lectures can have limited success in helping students acquire conceptual and procedural knowledge and can restrict them to basic formula-centred problem solving techniques $-{ }^{[6]}$; studies carried out on an introductory physics course indicate great improvements to learning and problem-solving expertise when students are provided with activities in which they become active participants using qualitative reasoning and concepts to solve problems. To become a more permanent part of their knowledge and learning, students need to apply their skills in a variety of different contexts over an extended time period.

It was decided to introduce a series of workshop sessions over a period of several weeks with the aim of increasing levels of learning and analytical thinking, tested by examination. These workshop sessions were integrated into the standard teaching scheme and did not introduce any additional hours to the timetable for staff or students. Students were encouraged to form co-operative groups for the sessions. The tasks set to the students were intended to be challenging and to improve general skills in Control, but 
also to encourage students to explore further areas that interested them. The workshop settings were kept informal with as little lecturer involvement as possible, relying heavily on student peer support. Presentations by the lecturer lasted no longer than 15 minutes and were restricted to providing the students with an introduction to the problem context and an indication of areas to investigate.

\section{Method}

The students were presented with a plant to investigate and for development of a digital control loop. Real world industrial plants include a degree of uncertainty and variability, not typically present in teaching laboratory equipment. The plant chosen for this was a steam/water heat exchanger, previously installed in the engineering workshops. Prior to its removal as part of building refurbishments, the heat exchanger had been used for development of modelling and control strategies, which had been published in two journals. ${ }^{[7]},{ }^{[8]}$.

The first paper ${ }^{[7]}$ describes an automatic tuning method for the design of PID controllers and comprises accurate modelling and control design achieved by a combination of polezero cancellation and frequency response methods. The second paper ${ }^{[8]}$ describes the process of measuring the variance of the heat exchanger in real time enabling the application of adaptive statistical process control to supervise the continuous process loop. Each paper describes the plant in detail and presents the modelling that had been carried out.

Students were initially invited to investigate the two papers as a case study to familiarize themselves with the plant and model, the principle features of which are shown in Fig 1. Water was fed at a steady rate through the heat exchanger. The feed rate was controlled by a pneumatically operated valve as part of a simple flow control loop. The outlet temperature was controlled via the pneumatic steam valve. Steam was supplied at $10 \mathrm{psi}$ from a central boiler and as this also supplied steam to various users within the building such as process machinery in the polymer laboratory. Compressed air for the pneumatic valves was supplied from an accumulator which was pressurised by a central air compressor plant situated elsewhere in the building. The quality of the steam and the supply pressure varied with the number of other users on the steam main.. The heat exchanger had a dead-time of approximately $18 \mathrm{~s}$ and was characterised by a second order model with a major time constant of $15 \mathrm{~s}$, and the relationship between the water outlet temperature and the steam valve actuation was approximated by:

$$
G_{c}(s)=\frac{0.33 e^{-18.5 s}}{134 s^{2}+18.5 s+1}
$$

An analogue anti-aliasing filter was used to filter the process output prior to conversion. This was a simple first order filter with a $0.25 \mathrm{~s}$ time constant.

In the simulation work described for the development of the adaptive statistical process control loop supervisor [8], a coloured noise signal had been added to the simulation control loop, as a signal was required that displayed similar characteristics to the noise present on the actual heat exchanger system, which appeared to be a noisy 'random walk'. This comprised many different components of various frequencies, including random noise from instrumentation, and cyclic variations from disturbances such as: changes in the steam pressure and quality from the boiler plant, changes in control air pressure, variations in the mains water temperature, variations in the ambient 
temperature, and variations in the water speed through the heat exchanger. To create a realistic noise component, white noise was integrated to produce a random walk and then the white noise was added back to the random walk. The resulting signal has a similar pattern to the noise observed on the heat exchanger plant and was considered to be a realistic representation of composite noise present on a typical industrial process subject to variability from numerous sources. Whist the creation of the noise model was not described in great detail, there was sufficient information to point students in the right direction if they had studied the papers sufficiently. Following an initial investigation of the plant model, students were invited to:

Develop a realistic digital-discrete model of the heat exchanger rig and demonstrate the performance

Develop and demonstrate accurate control of the temperature output.

The students were told that they were expected to answer questions on areas such as conversion methods, transport delay, realistic process noise simulation, programme loop details, and controller tuning.

The students were also told that:

For the purposes of the workshop it was acceptable to alter the continuous model deadtime and select one that simplified the discrete model so as not to introduce an excessive number of poles at the z-plane origin, e.g. use a lag that is twice the sample time, rather than the 18.5 seconds identified in the original published model.

The work was to be carried out using Matlab but the use of Simulink was not permitted. This was to ensure that they understood the discrete modelling and simulation techniques in preparation for their final examination

The students had a basic understanding of data manipulation and programme development in Matlab from work earlier in the year.

The students were encouraged to produce individual development work but to organise themselves into small co-operative groups of 3-4. The sessions were arranged in two hour blocks with a support tutor available by request during the hour following the sessions. Students were expected to be present for at least one hour of the two hours but were encouraged to take the lead in the development of their own work. Any formal presentations given by the lecturer were used to suggest further areas for development rather than to instruct students about how to carry out their work.

The students were told that there was no one correct answer to the tasks and were encouraged to explore. At the end of the four weeks of workshop sessions, students were required to present their work to the lecturer and answer questions. This formed part of the assessment for this unit. 


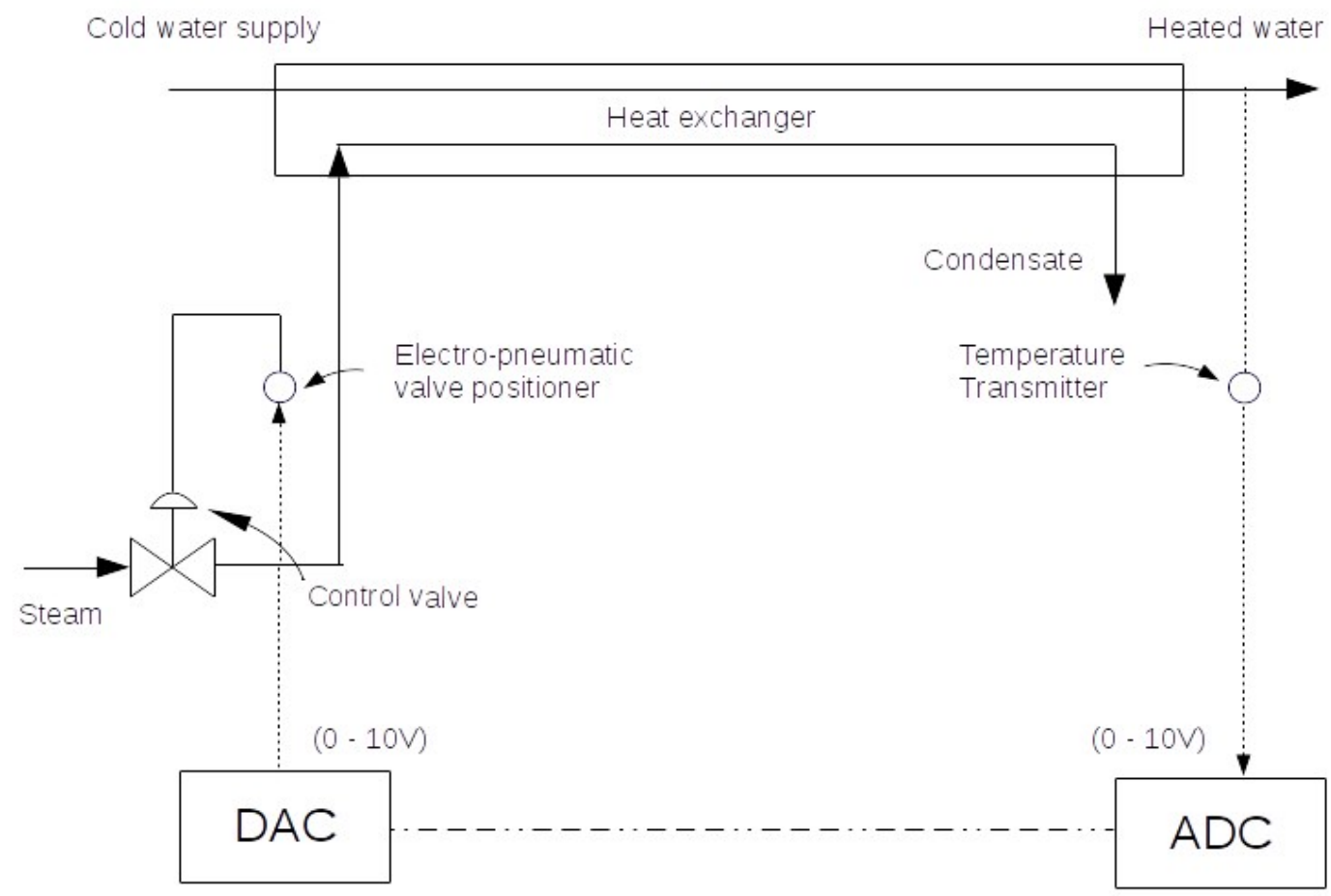

Figure 1 - Schematic image of heat exchanger

\section{Results}

The primary aim of the workshop exercises was not to produce high quality plant simulation and advanced control strategies, but to allow the students to explore and study challenging areas of discrete and continuous control in a relaxed and informal supportive environment. It would have been possible to guide the students much more intensively to produce sophisticated control schemes and models that performed much closer to the operation of the plant, but the strategy was for the workshop activities to be informal and student-led with an emphasis on exploration and experimentation. In many cases students greatly exceeded expectations in the level of the work. Some students surprised unit staff by producing work in areas not anticipated such as exploration of different models and control schemes, and comparison of different tuning methods for a robust performance. Teaching staff observed engagement amongst all students was much higher. As the workshops progressed, students were observed to have developed effective support networks, improving both communication and learning. Whilst attendance was only requested for one hour out of the two timetabled hours each week and the tutor's formal input was restricted, the sessions were very active and every student eligible for assessment opted to attend the workshop sessions. The approach was fully supported by management, particularly because there was no additional teaching load. As students were required to discuss their work and answer questions at the end of the four week of sessions, the individual development and contribution of each student could easily be 
established and this encouraged all students to participate in the learning process. As the workshops progressed, students were observed to develop effective support networks, improving communication and learning.

Figure 2 shows a plot of the original heat exchanger temperature outlet under PID control. The noise profile appears to comprise of random noise with a superimposed slower periodic variation of around 400s. Data analysis of the temperature output indicated that the noise component was approximately 5\% and consisted of coloured (random walk) noise plus white noise. The control air and heating supplies, resulted in air pressure and steam quality that were subject to cyclic and random variations which are thought to be the cause of the lower frequency noise observed.

Figures $3 \mathrm{a}$ and $3 \mathrm{~b}$ show examples of two students' work. In each case the plant and noise has been modelled and subject to a step input of $10^{\circ} \mathrm{C}$. In the first of these two, fig $3 \mathrm{a}$, the steady state temperature has a higher white noise component. In the second of these two, fig $3 \mathrm{~b}$, the steady state temperature has a higher random walk component with less white noise. Figure 4 is an example of a student's work showing the response to a simulation of the plant regulated by a PID controller subject to a step input of $50^{\circ} \mathrm{C}$; the student was experimenting with different tuning strategies. Such experimentation in a relaxed environment greatly contributes to the learning process, and staff observed a much higher level of understanding of control strategies following the series of workshop sessions. Development of the noise model for the heat exchanger provided students with a simple introductory task to experiment with converting difference equations in to discrete loops. Overall students were observed to develop skills significantly in the areas of discrete system skills, and continuous control strategies, which form the major part of the final exam in this unit.

In the five years preceding the introduction of the workshop study exercises, the average exam mark had been $52.5 \%$. In the year the workshop study was introduced, 2013-14, the exam mark was $65.3 \%$ and in for the $2014-15$ intake the exam mark was $68.8 \%$. No other alterations were made to the syllabus or exam preparation besides the introduction of the workshop sessions. Figure 5 shows the average examination percentage for each class from years 2009/10 to 2014/15. The improvement to the student exam results can clearly be seen as the workshop sessions were introduced in 2013/14.

Student comments from years 2013/14 and 2014/15 included:

'this is one of the best things we have done on the course.'

'I really feel ready for the final exam now'

'the fear has been taken away from this subject and now I can get on with learning it'

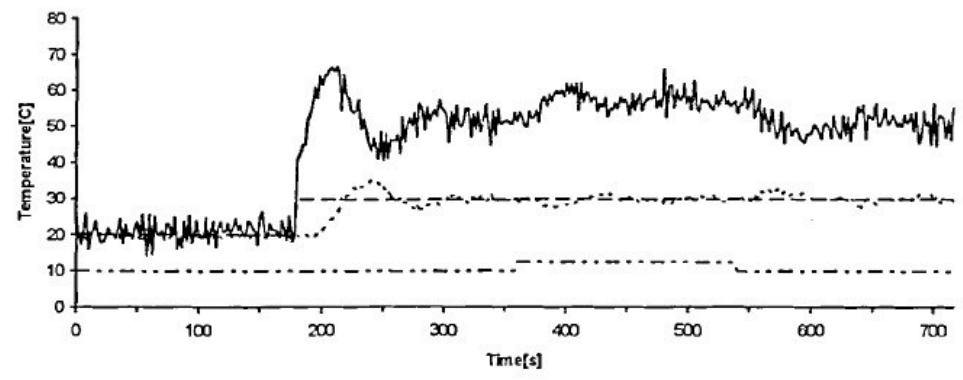

Figure 2 - Original graph of temperature outlet of heat exchanger under PID control 


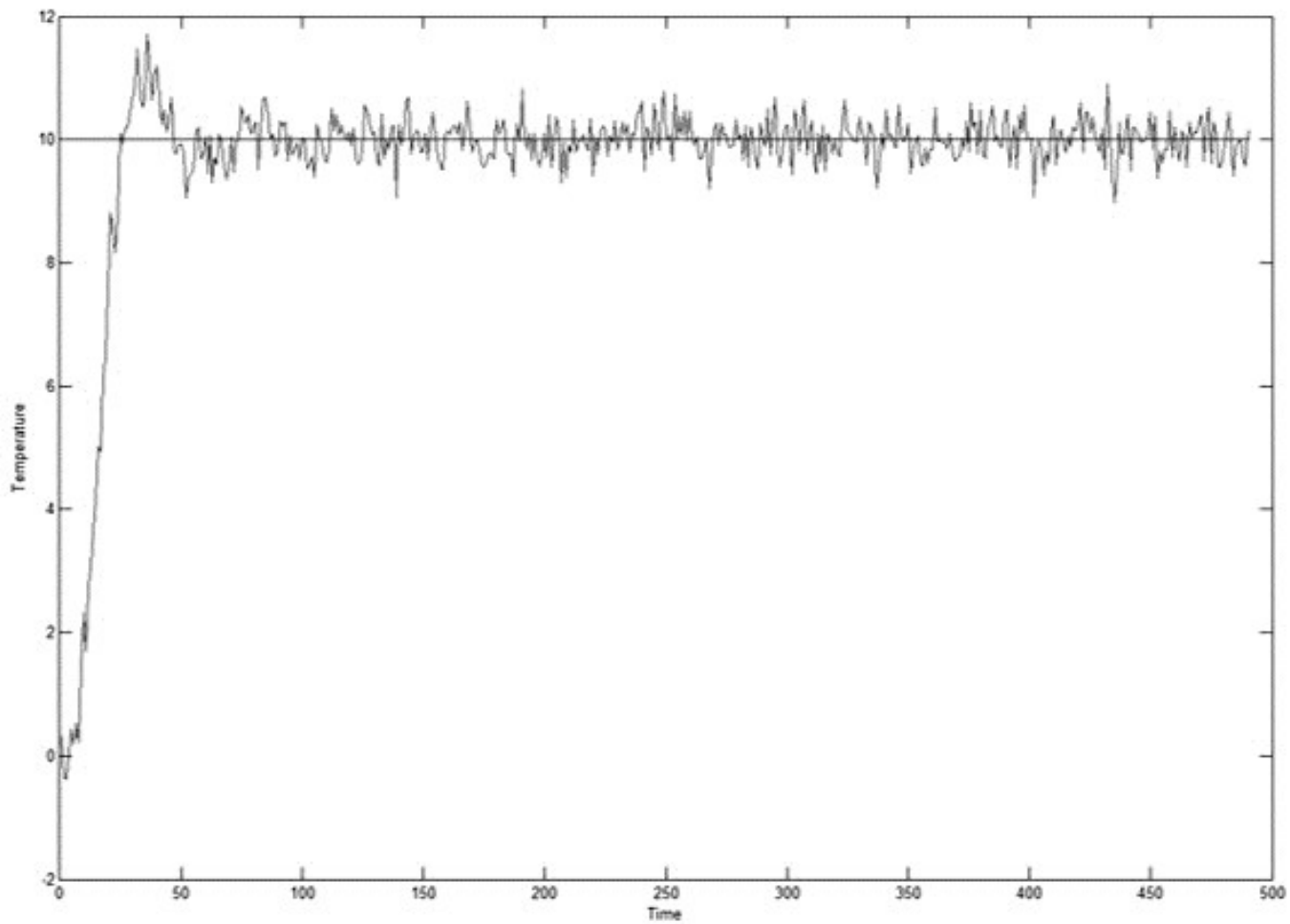

Figure $3 \mathrm{a}-$ Simulation response of the temperature outlet from student work (A) of heat exchanger 


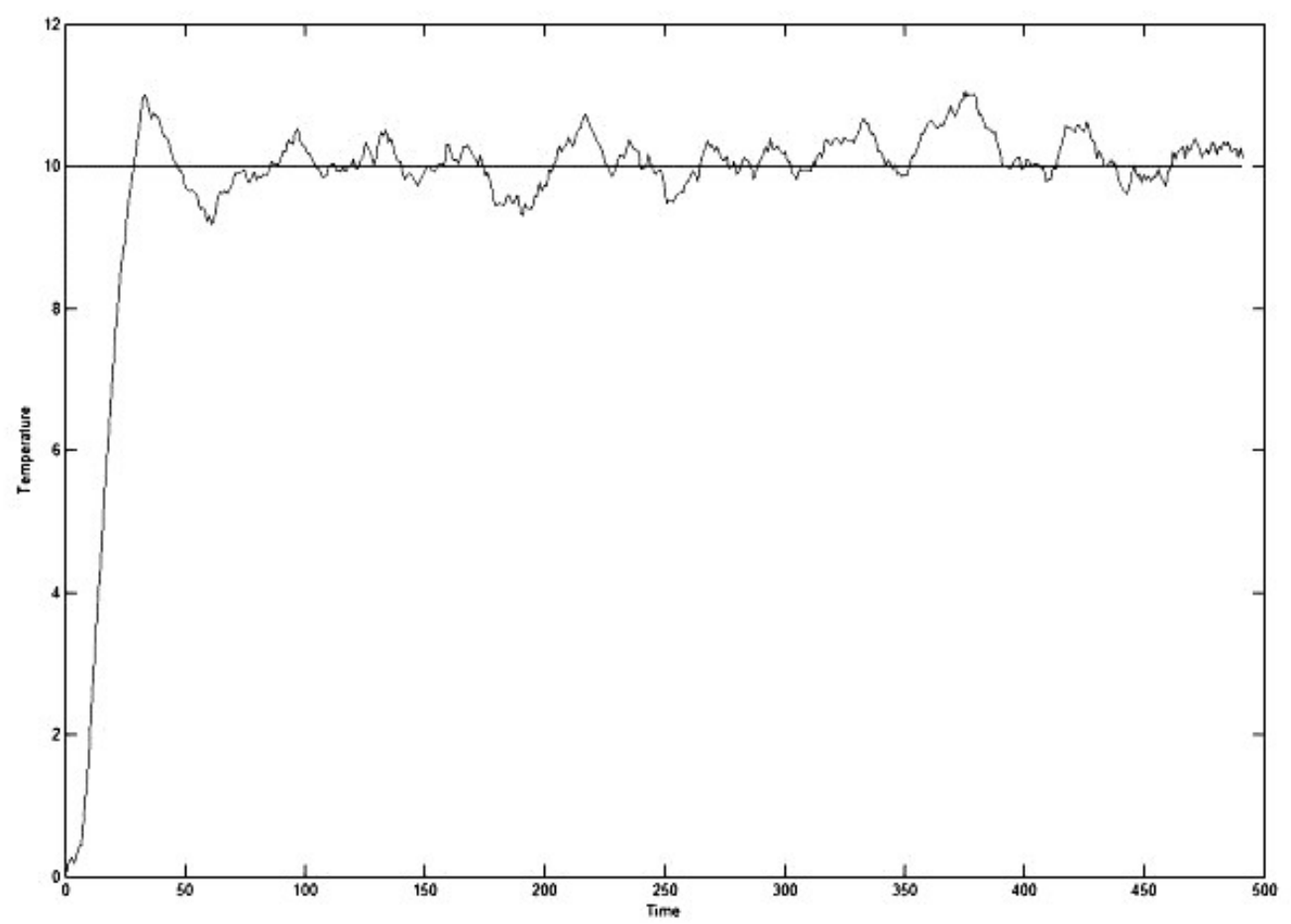

Figure $3 \mathrm{~b}$ - Simulation response from student work (B) of heat exchanger using coloured noise added to control loop

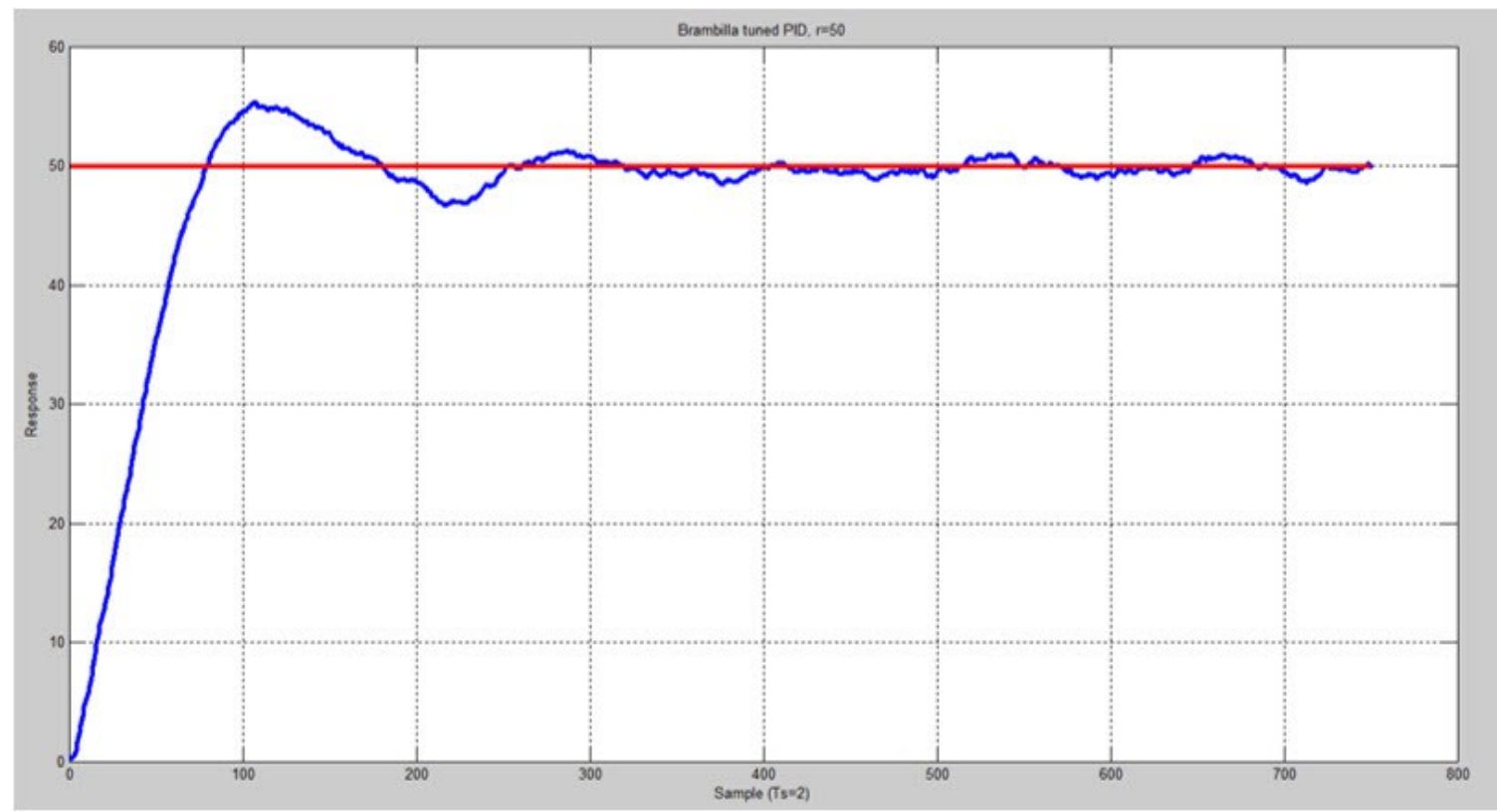

Figure 4 - Simulation response from student work of modulated heat exchanger investigating Brambilla tuning 


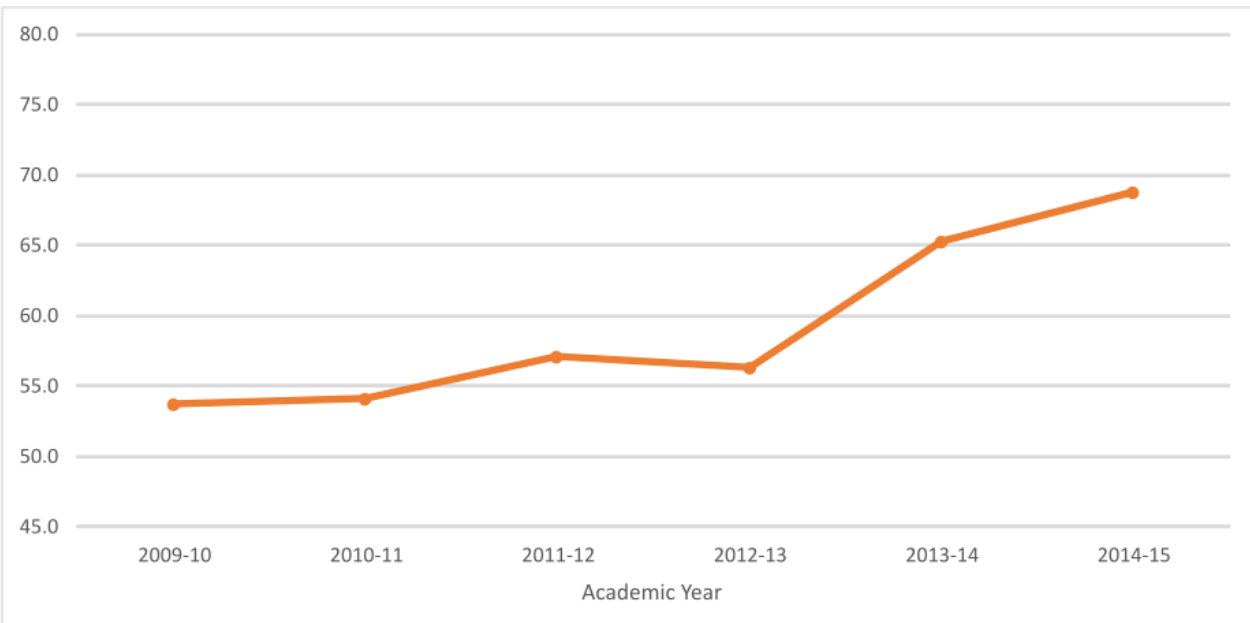

Figure 5 - exam percentage average each year

\section{Discussion}

Lecturers of the final year unit teaching digital and continuous control were prompted to change the teaching strategy following concerns raised by students about the apparent difficulty in passing the unit and because of the relatively low average pass mark for the final exam at 52.5\% over years between 2008/09 and 2012/13. Previously increased tutorial support had not created any measurable improvements.

The workshop sessions were introduced as a trial in an attempt to improve learning and student satisfaction. The strategy was for the sessions to be student led, informal and challenging, encouraging exploration of more advanced control concepts. No additional timetabled sessions were introduced and the sessions had to be integral to the teaching scheme rather than elective.

The sessions were very active and attendance was virtually $100 \%$. Once the workshop activities were well designed and planned, implementation of them was straightforward and did not cause any additional work for unit staff. Exam marks improved considerably in the two years following introduction of the workshop sessions. Feedback and enthusiasm from the students also improved considerably.

\section{Acknowledgements}

The authors would like to thank the School of Engineering, Manchester Metropolitan University, UK for internal support received during the development of the workshops, and during the writing/reviewing of the manuscript.

\section{Funding}


The authors received no financial support for the research, authorship, and/or publication of this article.

\section{References}

[1] R. Preszler, 'Replacing Lectures? with Peer-led Workshops Improves Student Learning', CBE - Life Sciences Education, 8 , (2009). DOI: 10.1187/cbe.09-01-0002

[2] J. K. Knight and W. G. Wood, 'Teaching More by Lecturing Less', Cell Biol. Educ., 4 (2005). DOI: 10.1187/05-06-0082

[3] - L. T. Tien, V. Roth, J.A. Kampmeier, 'Implementation of a Peer-Led Team Learning Instructional Approach in an Undergraduate Organic Chemistry Course', Journal of Research in Science Teaching, 39, (2002). DOI 10.1002/tea.10038

[4] - R. W. Preszler, 'Student- and Teacher-Centered Learning in a Supplemental Learning Biology Course' Student and Teacher-Centered Bioscene, 32, (2006).

[5] D. R. Arendale, 'Pathways of Persistence: A Review of Postsecondary Peer Cooperative Learning Programs' in Best Practices for Access and Retention in Higher Education, Center for Research on Developmental Education and Urban Literacy, General College, University of Minnesota, (2004).

[6] A. V. Heuvelen, 'Learning to Think Like a Physicist: A Review of Research-based Instructional Strategies', Am. J. Phys. 59, (1991).

[7] M. Thomson, P. G. Cassidy, D. J. Sandoz, 'Automatic tuning of PID controllers using a combined time- and frequency-domain method', Trans Inst Measurement and Control, 11, (1989). DOI: 10.1177/014233128901100105

[8] P. Twigg and M. Thomson, 'On-Line Noise Estimation for Adaptive SPC Loop Control', Trans Inst Measurement and Control, 17, (1995). DOI:

$10.1177 / 014233129501700303$ 
